\title{
Haptizing Wind on a Weather Map with Reactive Force and Vibration
}

\author{
Masaki Omata ${ }^{1}$, Masami Ishihara ${ }^{2}$, Misa Grace Kwok ${ }^{1}$, and Atsumi Imamiya ${ }^{1}$ \\ ${ }^{1}$ Interdisciplinary Graduate School of Medicine and Engineering, \\ University of Yamanashi, 400-8510 Kofu, Japan \\ \{omata, grace, imamiya\}@hci.media.yamanashi.ac.jp \\ ${ }^{2}$ Graduate School of Engineering, University of Yamanashi, \\ 400-8510 Kofu, Japan \\ masami@hci.media.yamanashi.ac.jp
}

\begin{abstract}
This paper describes a model for haptizing wind on a weather map. To design the model, we examined the human sensory scale to represent wind speed and direction with appropriate haptic stimuli, and examined parameters of the stimulus that allow a user to easily recognize changes in wind speed and direction. The results of these experiments show that vibration frequency can represent wind speed while a constant reactive force represents direction. The model solves a problem users of reactive force-only systems have difficulty identifying direction when the force is small due to light wind. Based on the model, we have developed a prototype weather information system with visual and haptic information.
\end{abstract}

\section{Introduction}

Haptic stimuli are used to present complex scientific information, such as hydrodynamic and weather data, in a readily understandable format. Techniques for haptizing generally provide the fingertips or palms of the user with tactile stimuli by using a kinesthetic feedback device [1].

One application of this technique is haptizing wind. This allows the user to feel wind speed and direction by assigning a reactive force or vibration to these properties $[2,3]$. However, in previous systems, which convey direction and speed using the same reactive force, users have had difficulty perceiving direction when wind speed is low, just as with real wind.

To address this problem, we investigate using separate tactile stimuli to represent wind speed and direction. This requires identifying two stimuli that users can perceive changes in simultaneously and without confusion.

In this study, we examined variations in several tactile stimuli and concluded that vibration frequency can effectively represent wind speed while a constant reactive force represents direction. We then apply this combination to a model of haptization.

\section{Related Works}

Kashiwabara et al. suggested a technique for visualization and haptization of swirling flow in a pipe, implementing contact sensation of virtual particles [2]. The technique 
converts velocity and pressure vectors into reactive forces in order to produce haptic data from particle distribution data.

Reimersdahl et al. developed ViSTA FlowLib, which provides haptic information about fluid motion for cases where it is difficult to represent the motion visually, such as local fluid changes [3]. This system provides a scalar field and a vector field of tactile sensation.

A problem with these approaches is that, in faithfully reproducing the relative magnitudes of the variables, they do not consider limitations in the users' sensitivity to tactile stimuli. However, some studies have adapted human sensory scales into haptization. Noma et al. studied a method of representation of volume data using a forcefeedback display with haptic sensation [4]. This system uses a reactive force and twisting torque with six degrees of freedom and provides visual information through a head-mounted display. They adjusted the intensity of tactile stimuli to human perception using a difference threshold. However, their system has not been experimentally validated.

Yano et al. developed the VibroGlove and proposed using it to haptize through vibration the direction and speed of a fluid stream [5]. The VibroGlove is a CyberGlove (Virtual Technologies Inc.) mounted with seventeen vibrators. They designed a haptic system based on human perception by correcting the intensity of tactile stimuli using magnitude estimation, sensory accuracy perception, and a difference threshold of vector directions. The VibroGlove has been used to represent the overall flow field of large spaces, such as CAVE [6]. However, it is not clear if it can be used for small spaces, such as PHANToM (SensAble Technologies Inc.). It is still necessary to determine the optimal tactile stimulus type and parameters for haptizing flow at a single point, such as a stylus or fingertip.

\section{Perception Experiments with Haptized Wind}

This section describes perception experiments to address the problem of haptizing wind. We examined various tactile stimuli to represent wind speed and direction, with consideration of human sensory characteristics and sensitivities. We empirically investigated combined tactile stimuli to simultaneously convey wind speed and direction. We used a two-dimensional vector field on a weather map, employing the Beaufort wind scale for representing speed with sixteen possible directions.

\subsection{Experimental Environment}

We used PHANToM and a Reachin Display（Reachin Technologies AB） for haptizing wind (Fig. 1). PHANToM provides a stylus with force feedback, allowing a user to feel reactive force, vibration, hardness, roughness, slope and salience. The Reachin system reflects three-dimensional graphics off a semitransparent mirror, so that they appear below the plane of the mirror. The user can see his hands underneath the mirror and "touch" the virtual objects. We presented overall information visually, and local detailed information haptically. The devices were connected to a Windows PC (Intel Pentium III $700 \mathrm{MHz}$ dual CPU, $512 \mathrm{MB}$ RAM, INTERGRAPH Intense3D 4105 graphics card, Microsoft Windows 2000 Professional OS). We used Reachin 
API 3.0 to create a three-dimensional space and to develop tactile stimuli for representing wind.

In a subject's differentiation between tactile stimuli is confounded by PHANToM's motor, which produces distinguishable noises. Therefore, subjects wore soundmasking headphones (Fig. 1.).

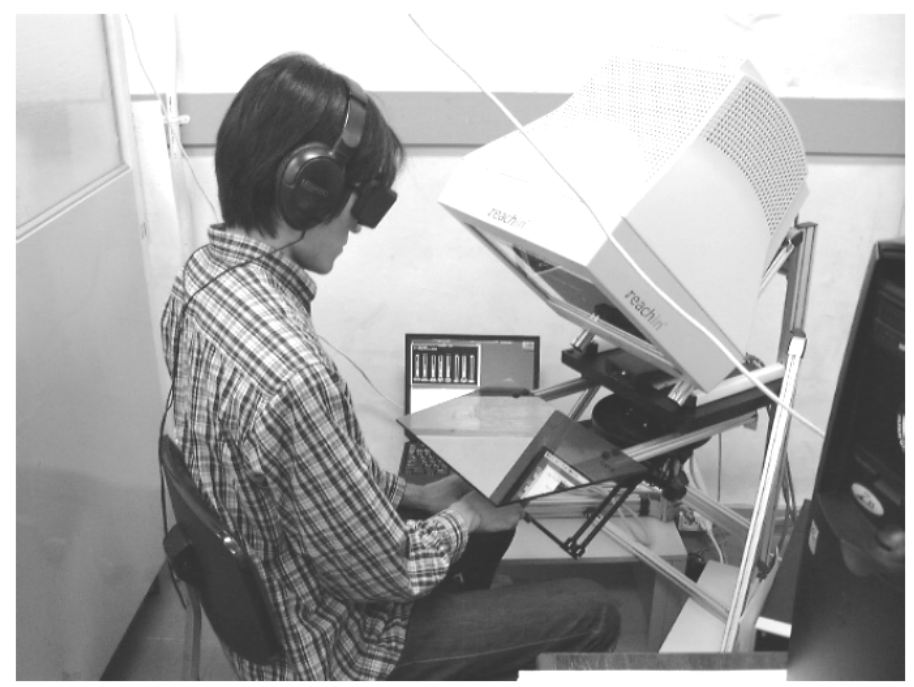

Fig. 1. Experimental environment with PHANToM and Reachin Display

\subsection{Appropriateness of Stimuli}

We asked subjects to compare the appropriateness of four stimuli for wind flow field, using Scheffé's paired-comparison method. The stimuli tested were those which PHANToM could generate and users could feel changes in without moving the stylus or having to repeatedly "touch" a surface.

Experimental Task. A Subject put the top of stylus on a plate (the size is $5 \mathrm{~cm} \times 6 \mathrm{~cm}$ ) and answered appropriateness of a tactile stimulation to wind. Fig. 2 shows six plates assigned two tactile stimulations on the half mirror of Reachin Display. Three of the six plates were placed on upper side, and other three of them were placed lower side. The three plates on upper side were assigned one tactile stimulation of four stimulations, and the three plates on lower side were assigned another tactile stimulation. Moreover, each plate was assigned the different intensity of stimulus.

A subject answered appropriateness between two stimulations on each side separately after he/she touched the six plates about five seconds of each other. While touching the plates, the subject pointed at the same position on the plate without tracing the plate.

We used a 7 point Likert scale as an answer of a trial. A subject, therefore, needed to select one of the 7 points between two tactile stimulations. We also used Scheffé's paired comparison method to analyze subjects' answers between two stimulations. 


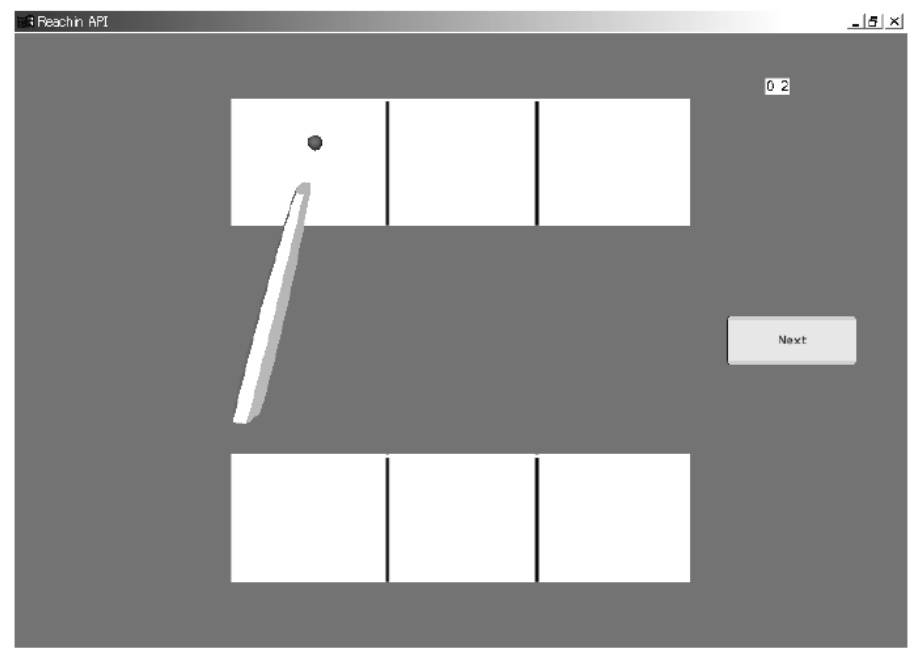

Fig. 2. Display for evaluation for appropriateness

Experimental Stimuli. Fig. 3 and Table 1 show four tactile stimulations of our experiment, which are reactive force, vibration, hardness and slope. Reactive force $(0.5$, 1.0, $2.0[\mathrm{~N}]$ ) is unidirectional force to push the stylus form right to left on a parallel with a plate. Vibration (frequencies: $0.5,1.0,1.5[\mathrm{~Hz}]$, amplitude: $1.0[\mathrm{~N}]$ ) is simple harmonic oscillation to move the stylus longitudinally on a parallel with a plate. Hardness $(50,100,200[\mathrm{~N} / \mathrm{m}])$ is normal force on a plate when a subject vertically pushes the stylus on a plate. Slope $(0.5$ [rad]) is slope of a plate that rotates on its center axis, which divide the plate in half longitudinally. As regarding the slope stimulation, we set the just one condition because we thought that to change an angle of the slope had no effect on subjects when the subjects pointed at the same position on a plate.

The color and the size of all plates are the same each other as visual information, and the differences among the plates are just tactile stimulation as haptic information.

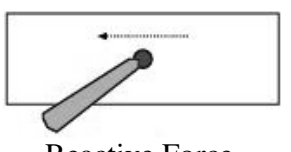

Reactive Force

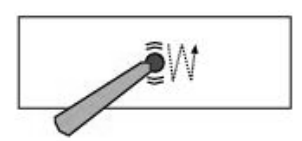

Vibration

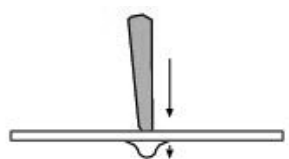

Hardness

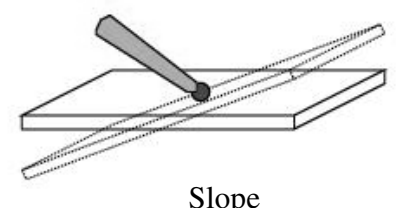

Slope

Fig. 3. Four tactile stimuli for evaluation for appropriateness 
Table 1. Intensities of four tactile stimuli

\begin{tabular}{l|l}
\hline \multicolumn{1}{c|}{ Stimulus } & \multicolumn{1}{|c}{ Intensities } \\
\hline Reactive force & $0.5 \mathrm{~N}, 1.0 \mathrm{~N}, 2.0 \mathrm{~N}$ \\
Vibration & $0.5 \mathrm{~Hz}, 1.0 \mathrm{~Hz}, 1.5 \mathrm{~Hz}$ \\
& Amplitude: $1.0 \mathrm{~N}$ \\
Hardness & $50 \mathrm{~N} / \mathrm{m}, 100 \mathrm{~N} / \mathrm{m}, 200 \mathrm{~N} / \mathrm{m}$ \\
Slope & $0.5 \mathrm{rad}$ \\
\hline
\end{tabular}

Results and Analysis. Twenty right-handed subjects participated in this experiment. They consisted of fourteen males and six females between 26 and 30 years old.

The main effect of appropriateness among four stimulations is significantly different by ANOVA $(\mathrm{p}<0.01)$. Fig. 4 shows psychological scaling of appropriateness among the four tactile stimulations about wind. In this figure, a higher level of the value of the scaling means a higher level of appropriateness about wind, and Y(0.05) and $\mathrm{Y}(0.01)$ mean a level of statistical significance between a stimulation and another one.

The analysis shows that reactive force and vibration are equivalent appropriateness about wind. In other words, we can use not only reactive force, which are used in conventional haptization, but also vibration to represent wind with haptic information. Namely, the result and analysis indicate possibility of a new haptization design that allows us to use reactive force to express wind power and to use vibration to express wind direction. Moreover, the design solves the problem that makes it hard to recognize a wind direction when the wind power is low.
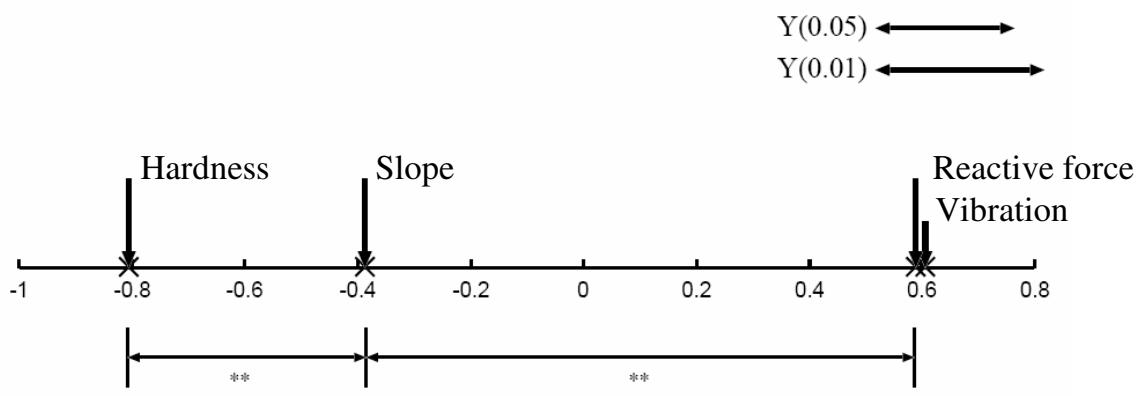

Fig. 4. Psychological scaling of appropriateness for representing wind. A higher level of the scaling means a higher level of appropriateness about wind.

\subsection{A Correspondence Examination Between Scale of Wind and Frequency of Vibration}

As discussed previously, we revealed that we can represent scale of wind by using change of frequency of vibration. For the next phase, we, therefore, established a model of sensory scales of correspondence between the scale of wind and magnitude of the frequency change. For this purpose, we conducted an experiment to determine 
the magnitude corresponding to scale of wind represented by numeric value and text as weather information. In this experiment, we used magnitude of the frequency change of wind in formula 1 (contribution ratio: 0.88 ) that was examined in a prior experiment. The reason why we used this formula is that the rule is Stevens' power law between physical quantity $(I)$ and haptic sensation ( $I$ ) [7]. In formula 1, $I$ means physical value, and $k$ and $\alpha$ mean constant numbers, which were examined in the prior experiment.

$$
I=\left(\frac{S}{k}\right)^{\frac{1}{\alpha}}
$$

(coefficient: $k=5.20, \alpha=1.26$, contribution ratio: 0.88 )

Experimental Task. A square plate $(20 \mathrm{~cm}$ on a side) was represented on a half mirror of the Reachin Display (Fig. 5). Numeric value of force of wind and text that explains a situation of wind was displayed on the square (in Japanese). Subjects read the value and text with touching the plate, which was assigned vibration, and adjusted magnitude of the frequency change to his/her sense of the wind by using "Up" button to increase the frequency and "Dn" button to decrease the frequency. Before a subject started performing a task, he/she identified the upper frequency limit and the lower frequency limit, which were adjustable range of a frequency. In addition, we set a frequency randomly in the range when a subject started performing a task.

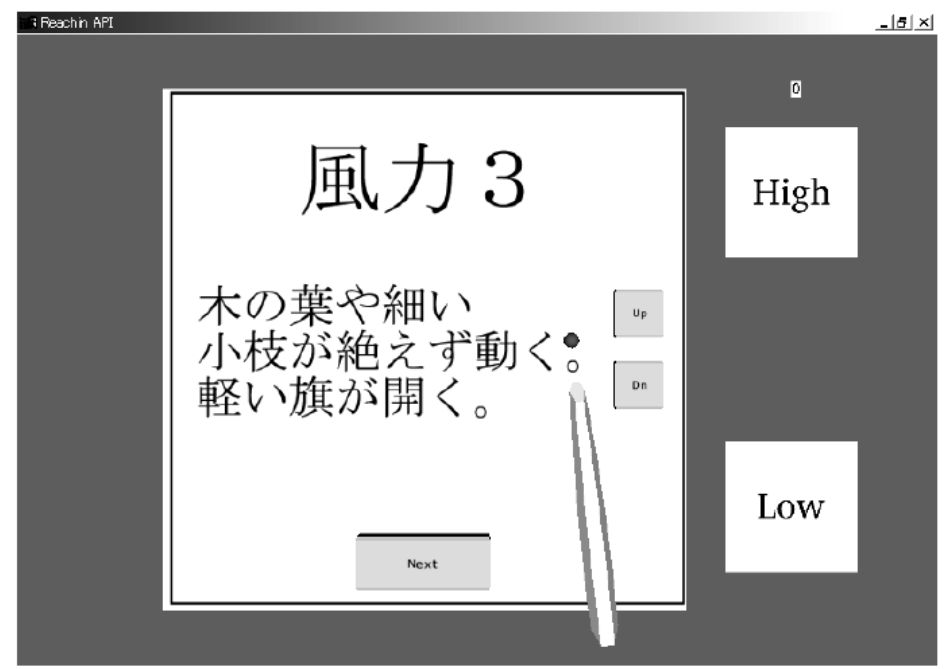

Fig. 5. Display for adjusting vibration frequency to wind speed 
Experimental Stimuli. We used the Beaufort wind scale of The Japan Meteorological Agency as value of force of wind and text that represent a state of wind power. The wind scale consists of thirteen degrees of value from 0 to 12 and specification about human activities about wind on land (Table 2) [8] [9]. Amplitude of vibration was $0.83[\mathrm{~N}]$ established in a prior experiment. The $0.83[\mathrm{~N}]$ is the lower limit to recognize a vibration with PHANToM. Direction of a vibration was lengthwise direction of the square, as in the previous experiment (section 3.2).

Table 2. Beaufort wind scale [8]

\begin{tabular}{|c|l|}
\hline Force & \multicolumn{1}{|c|}{ Description } \\
\hline 0 & Calm; smoke rises vertically. \\
\hline 1 & $\begin{array}{l}\text { Direction of wind shown by smoke drift, but not by wind } \\
\text { vanes. }\end{array}$ \\
\hline 2 & Wind felt on face; leaves rustle; ordinary vanes moved by wind. \\
\hline 3 & $\begin{array}{l}\text { Leaves and small twigs in constant motion; wind extends light } \\
\text { flag. }\end{array}$ \\
\hline 4 & Raises dust and loose paper; small branches are moved. \\
\hline 5 & $\begin{array}{l}\text { Small trees in leaf begin to sway; crested wavelets form on } \\
\text { inland waters. }\end{array}$ \\
\hline 6 & $\begin{array}{l}\text { Large branches in motion; whistling heard in telegraph wires; } \\
\text { umbrellas used with difficulty. }\end{array}$ \\
\hline 7 & $\begin{array}{l}\text { Whole trees in motion; inconvenience felt when walking against } \\
\text { the wind. }\end{array}$ \\
\hline 8 & Breaks twigs off trees; generally impedes progress. \\
\hline 9 & $\begin{array}{l}\text { Slight structural damage occurs (chimney-pots and slates re- } \\
\text { moved). }\end{array}$ \\
\hline 10 & $\begin{array}{l}\text { Seldom experienced inland; trees uprooted; considerable struc- } \\
\text { tural damage occurs. }\end{array}$ \\
\hline 11 & Very rarely experienced; accompanied by wide-spread damage. \\
\hline 12 & Very rarely experienced \\
\hline
\end{tabular}

Results and Analysis. Twenty-two right-handed subjects participated in this experiment. They consisted of sixteen males and six females between 20 and 24 years old.

Fig. 6 shows a boxplot that illustrates a correspondence between force of wind (horizontal axis) and subjects' sensation magnitude for frequency of vibration (vertical axis). A whisker on the boxplot means upper inner fence (a farthest value of 75 th percentile) or lower inner fence (a farthest value of 25th percentile), and a halfway line represents median. An open circle on the boxplot corresponds to outliers.

The result shows that median of the sensation magnitude linearly increases with increasing value of force of wind. Therefore, the correspondence between force of wind $(W)$ and the sensation magnitude $(S)$ is illustrated by formula 2 , which is an approximated line with least-square method.

$$
S=a W+b
$$

(coefficient: $a=10.25, b=-2.25$, contribution ratio: 0.97 ) 


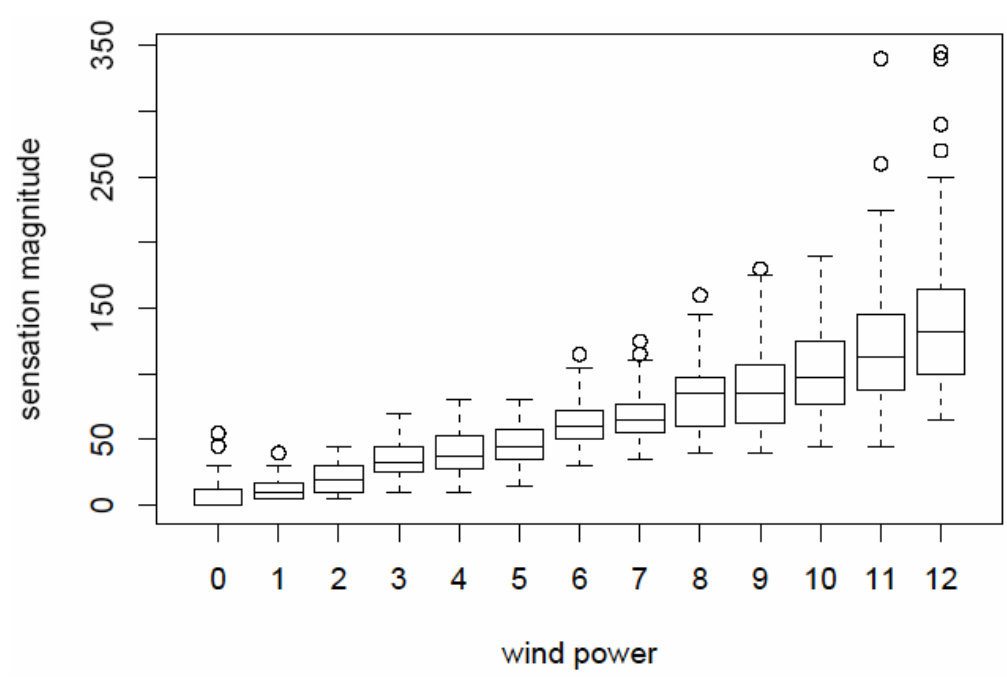

Fig. 6. Vibration frequencies corresponding to wind speeds

\subsection{Discrimination of Wind Direction with Reactive Force and Vibration}

In section 3.2 we established that it was possible to represent a wind flow field by either reactive force or vibration, and in section 3.2 we established a model of the vibration frequency used to represent wind speed. In this experiment, we investigated subjects' discrimination of wind direction on a wind flow field by representing wind speed by vibration frequency and wind direction by the direction of a constant reactive force. We then compared this system to one using a reactive force to represent both wind properties.

Experimental Task. Subjects were shown a 16-point compass rose on the Reachin Display (Fig. 7). Touching a direction with the stylus produced a randomly directed reactive force. Subjects were asked to push the button corresponding to the direction of the force.

Experimental Stimuli. There were two experimental conditions: one combining a reactive force with vibration, and the other just a reactive force. The vibration amplitude was $0.80[\mathrm{~N}]$ as described in the section 3.3, and the vibration frequency was $10[\mathrm{~Hz}]$, which was the mean value selected by subjects in the experiment of section 3.3. The reactive force was a constant $2.26[\mathrm{~N}]$, which was established in a prior experiment as the minimum constant force detectable using PHANToM. Directions for reactive forces corresponded to the 16 cardinal wind directions (e.g., north-northwest, south-southeast, etc.).

Results and Analysis. Nine right-handed subjects participated in this experiment. They consisted of six males and three females between 21 and 24 years old.

There was no significant difference between the two conditions in either average time to select a direction $(5.20$ [s] with vibration vs. 5.95 [s] without vibration) or the percentage of correctly identified directions $(53.56 \%$ with vibration vs. $51.85 \%$ without vibration). 


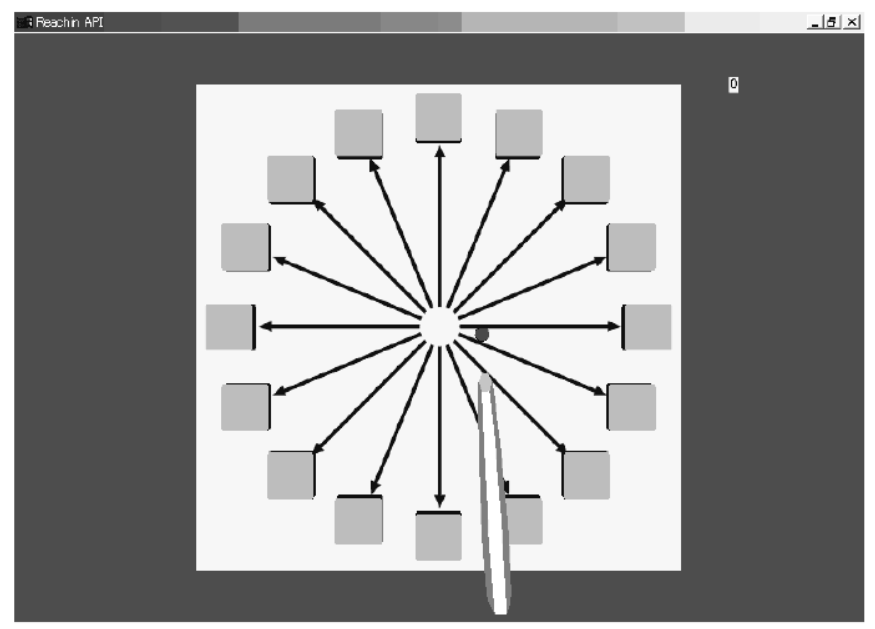

Fig. 7. Display for direction discrimination

The results show that it is difficult for subjects to discriminate among 16 directions for a reactive force. However, when subjects' responses were recalculated using only eight directions, the percentage of directions correctly identified in both conditions was over $90 \%$. Therefore, to allow users a high level of discrimination, our model must be limited to eight wind directions.

\section{A Model for Haptizing Wind Using Reactive Force and Vibration}

In this section, we propose a model for haptizing wind and a weather information system based on the model.

\subsection{A Model for Haptizing Wind with Consideration of Human Sensory Characteristics}

Our model for haptizing wind considers human sensory characteristics and sensory scales based on the results of the experiments described above. We limited the wind field to two dimensions, both to simplify evaluation of the model and to simplify integration of visual information for a weather information system.

Formula 3 represents each vector of wind on a grid flow field. In this formula, $v_{x}$ is vector quantity from east to west and $v_{y}$ is vector quantity from north to south.

$$
v=\left(v_{x}, v_{y}\right)
$$


In our model, wind speed is represented by vibration frequency and wind direction is represented by a constant reactive force. These are established in section 3.2.2.

Changes in vibration frequency are calculated from Formula 2. In addition, we define amplitude of vibration as a simple harmonic motion in order to avoid this force affecting the constant reactive force for wind direction. We use Formulas 4, 5 and 6 to generate simple harmonic motion. In Formulas 5 and $6, t$ is the time change and $f$ is the frequency of a simple circular harmonic motion.

$$
\begin{aligned}
& P=(P x, P y) \\
& P_{x}=0.80 \sin (2 \pi f t) \\
& P_{y}=0.80 \cos (2 \pi f t)
\end{aligned}
$$

Formula 7 is the constant reactive force that represents just a wind direction; it is composed of a unit vector and lower limit of detectable force. $u=(\sin \theta, \cos \theta)$ is a unit vector that is digitized from a wind vector $v=\left(v_{x}, v_{y}\right)$ to one direction of eight compass directions.

$$
D=2.26 u
$$

Force $F$ is resultant force transmitted through the stylus to represent wind:

$$
F=P+D
$$

\subsection{A Prototype Weather Information System with Visual and Haptic Information}

Using this model, we have developed a weather information system with visual and haptic information. The system allows a user to simultaneously recognize speed and direction of wind by vibration and reactive force, and the system combines the advantages visualization and haptic information. For example, the system allows a user to obtain global information, such as clouds and isobaric lines, as visual information, and local information, such as wind speed and direction at a point, as haptic information. Fig. 8 shows a prototype of the system. When a user indicates a point on the weather map, he or she can feel the speed and direction of the wind at the point with reactive force and vibration, and can see clouds around the point.

We embedded a quantizer and a renderer in this system. The quantizer obtains quantified wind data from WMO DDB (World Meteorology Organization Distributed Data Base) via FTP [10]. The renderer creates a virtual scene with visual and haptic information by using our proposal model with the data from the quantizer. The renderer updates wind data and a virtual scene in response to user's request. The quantizer downloads the latest weather data from the internet in response to update request from the renderer. Fig. 9 shows the data flow from the WMO DDB to PHANToM and Reachin Display. 


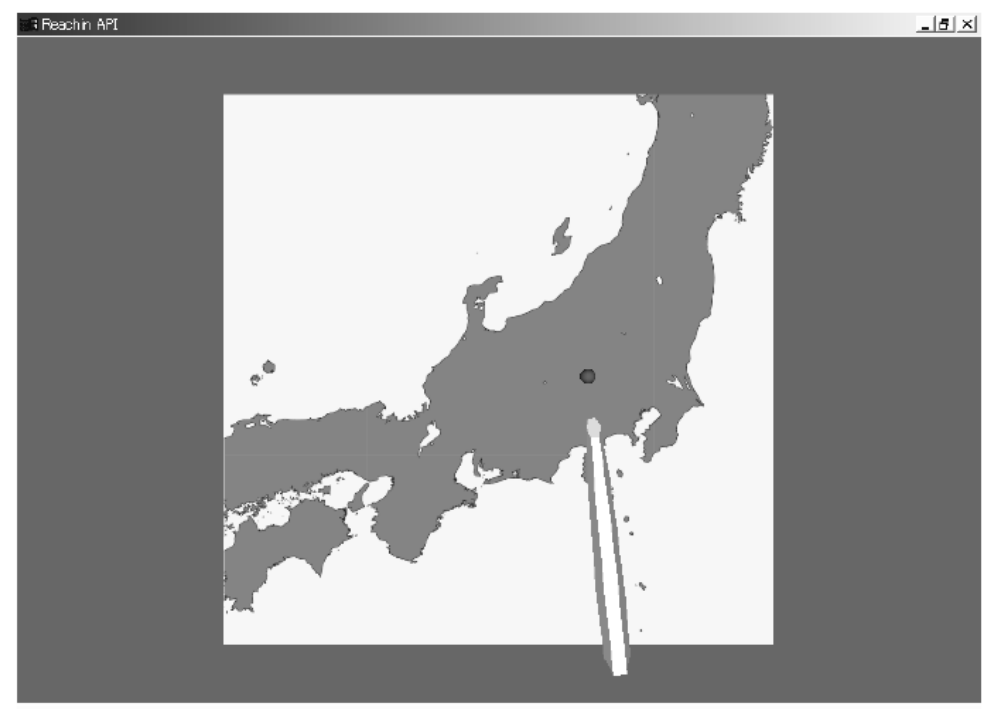

Fig. 8. Prototype weather information system with visual and haptic information

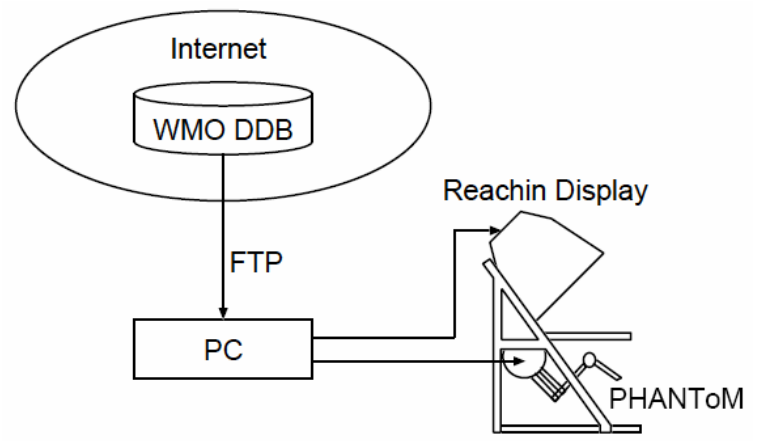

Fig. 9. Data flow diagram of a prototype weather information system

\section{Conclusions}

In this paper, we have examined a model for haptizing wind on a weather map that represents wind speed and direction simultaneously using different tactile stimuli, with a focus on selecting stimuli that are easy for the user to understand. To design the model, first we conducted experiments to determine appropriate tactile stimuli and intensities for representing wind. The results showed that vibration frequency can represent wind speed while constant reactive force represents wind direction. Our experiments also showed that users can reliably discriminate eight directions of a twodimensional wind flow field, even when reactive force and vibration are assigned to wind simultaneously. The model therefore solves the problem that users of reactive 
force-only systems have difficulty identifying direction when the force is small due to light wind.

Using this model we developed a prototype weather information system. The system provides a user with general weather conditions as visual information and wind speed and direction as haptic information. Therefore, this system incorporates a good part of features of both visual perception and tactile perception because users can put the weather in perspective through sight and can perceive a wind at a local point by the sense of touch without the distraction of visual information.

We hope to complete this system soon and then evaluate user interface about tactile stimulations based on our model. After that, we are planning to propose a new haptization model and a new haptic interaction based on human perception.

\section{References}

1. Massie, T.H., Salisbury, J.K.: The PHANToM Haptic Interface: A Device for Probing Virtual Objects. Proceedings of the ASME Winter Annual Meeting, Symposium on Haptic Interfaces for Virtual Environment and Teleoperator Systems, Chicago, 55-1 (1994) 295-300

2. Kashiwabara, S., Tanaka, Y., Suzuki, R.: Visualization and Haptization for Swirling Flow in a Pipe. Bulletin of Computational Science Research Center, Hosei University, Vol. 15, (in Japanese) (2002)

3. Reimersdahl, T., Bley, F., Kuhlen, T., Bischof, C.H.: Haptic Rendering Techniques for the Interactive Exploration of CFD Database in Virtual Environments, Proceedings of the Workshop on Virtual Environments (2003) 241-246

4. Noma, H., Iwata, H.: Presentation of Multiple-Dimensional Volume Data by 6 DOF Force Display, Progress in Human Interface, Vol. 3 (1994) 111-120

5. Yano, H. Hirose, M. Ogi, T., Tamura, Y.: Haptization of Flow Field Using Vibroglove. Transaction of Information Processing Society of Japan 40 (2), Special Issue on Human Interface and Interaction (in Japanese) (1999) 414-421

6. Cruz-Neira, C., Sandin, D. J., DeFanti, T. A.: Surround-Screen Projection-Based Virtual Reality: The Design and Implementation of the CAVE. Proceedings of SIGGRAPH (1993) 135-142

7. Lindsay, P. H., Norman, D.A.: Human information processing: An introduction to psychology (2nd Ed.). Academic Press (1977)

8. Asano, Y.: Juten Master Kisho-Yohoshi Shiken. Kobunsha (in Japanese) (2000)

9. National Weather Service. http://www.crh.noaa.gov/lot/webpage/beaufort

10. Japan Meteorological Agency, WMO Distributed Data Base /JMA2. http://ddb.kishou. go.jp/ 